\title{
A REMARK ON STRONGLY EXPOSING FUNCTIONALS
}

\author{
KA-SING LAU
}

\begin{abstract}
By using the concept of farthest points, we show that the set of strongly exposing functionals of a weakly compact convex subset in a Banach space $X$ is a dense $G_{\delta}$ in $X^{*}$. The construction also gives a new proof of existence of strongly exposed points in weakly compact convex sets.
\end{abstract}

Let $K$ be a convex subset in a Banach space $X$, a point $x \in K$ is called a strongly exposed point of $K$ if there exists an $f \in X^{*}$ such that (i) $f(x)>f(y)$ for all $y \neq x$ in $K$, (ii) for any sequence $\left(x_{n}\right)$ in $K$ with $f\left(x_{n}\right) \rightarrow f(x), x_{n} \rightarrow x$ in norm. We call the above $f$ a strongly exposing functional of $K$ and use $K^{\Lambda}$ to denote the set of strongly exposing functionals of $K$. Lindenstrauss [5] and Troyanski [6] proved that if $K$ is a weakly compact convex subset in $X$, then $K$ is the closed convex hull of its strongly exposed points. In [1], Anantharaman showed that if $K$ is the closed convex hull of the range of a vector-valued measure (hence $K$ is weakly compact) then $K^{\Lambda}$ is a dense $G_{\delta}$ in $X^{*}$. A similar conclusion has also been obtained by the author for weakly compact convex subsets in certain classes of Banach spaces [4]. In this note, by modifying the method in [4], we prove

THEOREM 1. Let $K$ be a weakly compact convex subset in a Banach space $X$; then $K^{\Lambda}$ is a dense $G_{\delta}$ in $X^{*}$.

In the proof, we will need the following propositions.

Proposition 2 (TroyANSKI). Let $X$ be a weakly compact generated Banach space; then $X$ admits an equivalent locally uniformly convex norm.

Proposition 3 (LAU). Let $K$ be a weakly compact subset in a Banach space $X$; then the set

$$
\{x \in X:\|x-z\|=\sup \{\|x-y\|: y \in K\} \text { for some } z \in K\}
$$

is a dense $G_{\delta}$ in $X$.

We call the point $z$ in the above proposition a farthest point of $K$ [2], [3]. It is known that if $X$ is locally uniformly convex, then a farthest point of a

Received by the editors September 16, 1975.

AMS (MOS) subject classifications (1970). Primary 46B99.

Key words and phrases. Banach spaces, farthest points, locally uniformly convex, strongly exposed points, strongly exposing functionals. 
bounded convex subset is also a strongly exposed point.

Proof of THE THEOREM. Note that

$$
\begin{aligned}
K^{\Lambda}=\bigcap_{n=1}^{\infty}\left\{f \in X^{*}: \operatorname{diam}\left\{x \in K: f(x)>\sup _{y \in K} f(y)-a\right\}\right. & \\
& \left.<\frac{1}{n} \text { for some } a>0\right\}
\end{aligned}
$$

and the set on the right side is a $G_{\delta}[1]$, [4]; hence it suffices to show the density of $K^{\Lambda}$ in $X^{*}$. By a remark in [4] and Proposition 2, we may assume that $X$ is weakly compact generated (say, by $K$ ) and locally uniformly convex. Let $f \in X^{*}$ with $\|f\|=1$. For $\varepsilon^{>0}$, let $C=f^{-1}(0) \cap 2 \varepsilon^{-1} B$ where $B$ is the closed unit ball of $X$. By a homothetic translation, we may let $K \subseteq B$ but $K \nsubseteq C$ (note that $K^{\Lambda}$ is unchanged). We will construct a point $z \in K$ which is a strongly exposed point of the closed set conv $(K \cup C)$. The corresponding strongly exposing functional $g$ of $\operatorname{conv}(K \cup C)$ with $\|g\|=1$ will satisfy $\|f-g\| \leqslant \varepsilon$ and also strongly exposes $K$ at $z$ (for details, cf. [4, Theorem 2.4]); hence this completes the proof.

Choose a point $x_{1} \in K \backslash C$ such that the set

$$
S=\left\{\alpha x_{1}+\beta y:|\alpha|^{2}+|\beta|^{2} \leqslant 1, y \in C\right\}
$$

does not contain $K$ (we neglect the case that $K$ is a singleton, $x_{1}$ may be chosen as midpoint of some line segment of $K$ not lying in $C$ ). Since $C$ is an absorbent subset of the hyperplane $f^{-1}(0), S$ is an absorbent subset of $X$. Let $\|\cdot\| \cdot \|$ be the norm defined by $S$; then $\|\cdot \cdot\|$ is locally uniformly convex and equivalent to the original norm. There exists $x_{2} \in K \backslash S$ with $\left\|x_{2}\right\| \|-1=\alpha>0$. By Proposition 3, there exist points $w \in X, z \in K$ with $\|w\| \leqslant \alpha / 2$ and $\beta=\|w-z\|$ $=\sup \{\|w-y \mid\|: y \in K\}$. For any point $y \in C$,

$$
\|y-w|\|\leqslant\| y \||+\alpha / 2 \leqslant 1+\alpha / 2<\beta .
$$

Hence $z$ is also a farthest point of conv $(K \cup C)$. It follows that $z$ is a strongly exposed point of conv $(K \cup C)$. Q.E.D.

We remark that the above construction yields another proof of the existence of strongly exposed points in weakly compact sets as in [5]. Moreover, we have

COROllary 4. Let $K$ be a weakly compact convex subset in a Banach space $X$; then for any bounded closed convex subset $C$ such that $K \nsubseteq C$, there exists a point $x \in K$ which strongly exposes conv $(K \cup C)$.

Proof. It follows easily from the above theorem and Theorem 2.4 in [4]: if $K$ is a bounded closed convex subset in $X$, then $K^{\Lambda}$ is a dense $G_{\delta}$ if and only if for any bounded closed convex subset $C$ such that $K \nsubseteq C$, there exists a point $x \in K$ which is a strongly exposed point of $\overline{\operatorname{conv}}(K \cup C)$. 


\section{REFERENCES}

1. R. Anantharaman, On exposed points of the range of a vector measure. II, Proc. Amer. Math. Soc. 55 (1976), 334-338.

2. M. Edelstein and J. E. Lewis, On exposed and farthest points in normed linear spaces, J. Austral. Math. Soc. 12 (1971), 301-308. MR 46 \#9694.

3. K. Lau, Farthest points in weakly compact sets, Israel J. Math. 22 (1975), 168-174.

4. - On strongly exposing functionals, J. Austral. Math. Soc. (to appear).

5. J. Lindenstrauss, On operators which attain their norm, Israel J. Math. 1 (1963), 139-148. MR $28 \# 3308$.

6. S. L. Troyanski, On locally uniformly convex and differentiable norms in certain non-separable Banach spaces, Studia Math. 37 (1970/71), 173-180. MR 46 \# 5995.

Department of Mathematics, University of Pittsburgh, Pittsburgh, Pennsylvania 15260 